\title{
Proceso de reflexión docente para mejorar las prácticas de evaluación de aprendizaje en el contexto de la educación para jóvenes y adultos (EPJA)
}

\author{
Teachers' Reflection Process to Improve Learning Assessment Practices \\ in the Context of Education for Youngsters and Adults (EPJA)
}

\author{
Processo de refleção docente para amelhorar as praticas de evaluação \\ do ensino no contexto da educação para jovens e adultos (EPJA)
}

\author{
Juan Ariel Muñoz Olivero ${ }^{1}$ \\ Carolina Pilar Villagra Bravo² \\ Segundo Edgardo Sepúlveda Silva ${ }^{3}$
}

Resumen

La educación para jóvenes y adultos (EPJA) ha alcanzado protagonismo en el sistema educacional chileno. Se ha ocupado de brindar una nueva oportunidad a un gran número de jóvenes que por una u otra razón no pudieron terminar sus estudios en la formación tradicional. En este escenario se indaga sobre las prácticas pedagógicas desarrolladas por los docentes que trabajan en la EPJA, especialmente las que se refieren a la evaluación de los aprendizajes. Por tanto, este trabajo tiene como objetivo presentar los resultados de un proceso de reflexión llevado a cabo por un grupo de docentes de un centro integrado de educación de adultos, con el fin de mejorar sus prácticas de evaluación del aprendizaje de sus estudiantes. El modelo de investigación se basa en el enfoque cualitativo con diseño de estudio de caso y se enmarca en un diagnóstico comprensivo de las prácticas, llevado a cabo a través de grupos de discusión. Una vez analizados los resultados del proceso, se concluye que la mayoría de estos docentes siguen prácticas evaluativas tradicionales, que tienden a replicar concepciones de evaluación centradas en la obtención de productos calificables para comprobar el logro, más que en la retroalimentación de procesos que potencien la autorregulación del aprendizaje. No obstante, la reflexión también deja ver que los docentes están dispuestos a participar en instancias de reflexión pedagógica que orienten la mejora de sus prácticas evaluativas.

Palabras clave: Reflexión pedagógica, práctica docente, evaluación del aprendizaje

Abstract

Education for youngsters and adults (in Spanish, EPJA) has reached prominence in the Chilean educational system. It has had to give a new chance to a large number of young people who, for one reason or another, did not complete their studies in traditional training. In this scenario, it explores pedagogical practices developed by teachers working with youngsters and adults, especially those who refer to learning assessment. Therefore, the purpose of this study is to present the results of a reflection carried out by a group of teachers from a comprehensive adult education center, in order to improve their practices to evaluate their students' learning. The research model is based on the qualitative approach with a case study design. It makes a comprehensive diagnostic of teaching practices, conducted through discussion groups. The analysis of the results shows that most teachers working at this educational center follow traditional assessment practices. They tend to copy conceptions of assessment centered around products that can be scored in order to check goal achievement, rather than around providing feedback that promotes self-regulated learning. However, the reflection also shows that teachers are willing to participate in pedagogical reflection sessions that guide them to improve their assessment practices.

Keywords: Pedagogical reflection, teaching practice, learning evaluation

3 Universidad Católica de Temuco, Chile. Correo electrónico: ssepulveda@uct.cl 
Universidad Pedagógica Nacional

Facultad de Humanidades

Resumo

A educação para jovens e adultos (EPJA) alcançou proeminência no sistema educacional chileno. Tem se-ocupando de proporcionar uma nova oportunidade para um grande número de jovens que, por alguma ração, não puderam concluir seus estudos na formação tradicional. Neste cenário se investigam as práticas pedagógicas desenvolvidas pelos professores que trabalham na educação de jovens e adultos, especialmente aquelas relacionadas com a avaliação da aprendizagem. Portanto, este artigo tem como objetivo apresentar os resultados de um processo de reflexão levada a cabo por um grupo de professores de uma educação de adultos integrada, a fim de melhorar suas praticas de evaluação do aprendizagem dos estudantes. 0 modelo de pesquisa baseia-se na abordagem qualitativa com desenho de estudo de caso e faz parte de umas práticas de diagnóstico abrangentes, conduzidas através de grupos de discussão. Depois de analisar os resultados do processo, conclui-se que a maioria destes professores seguiram as práticas de avaliação tradicionais, que tendem a replicar concepções de avaliação concentradas na obtenção de produtos qualificados para verificar o êxito, em vez de processos de retroalimentação que melhoram a auto-regulação da aprendizagem. No entanto, a reflexão também revela que os professores estão dispostos a participar de instâncias de reflexão pedagógica para orientar a melhoria de suas práticas de avaliação.

Palavras Chave: Refleção pedagogica, Pratica docente, Evaluação do aprendizagem

Artículo recibido el 10 de noviembre de 2014 y aprobado el 27 de enero de 2016

\section{Introducción}

La evaluación de los aprendizajes en el contexto educativo está claramente influenciada por dos paradigmas fundamentales: uno centrado en la medición y el resultado, en el que la evaluación se convierte en una comprobación del aprendizaje y un medio de control social; otro que plantea la evaluación como un proceso comprensivo y formativo cuyo resultado final es el aprendizaje propiamente dicho. Dentro de este marco, los docentes se ven enfrentados en su práctica en el aula a estas dos disyuntivas que, según sus creencias y desarrollo profesional, van matizando con el tiempo. Esta situación es transversal en todos los niveles educativos, en este caso en particular en la educación para jóvenes y adultos (EPJA).

En la actualidad la educación de adultos en Chile se está orientando a la educación de jóvenes, específicamente a una población cuyas realidades son complejas y diversas por sus historias personales, sus códigos culturales, sus experiencias educativas, su visión sobre el trabajo y participación en la sociedad. Esta situación ha generado un cambio de rol en la educación de adultos, ya que el actual desafío es incluir a estos jóvenes y ofrecerles nuevas oportunidades educativas, para lo cual, los docentes que atienden esta modalidad deben prepararse (Acuña, 2013).

Según el Ministerio de Educación (Mineduc), hacia el año 2011 la matrícula de jóvenes menores de 25 años inscritos en la modalidad regular de la EPJA bordeaba el 70\% y mostraba una tendencia al alza (Osorio, 2013). Estos jóvenes demandan soluciones específicas para su participación en esta modalidad, que implican cambios en relación con horarios, métodos de trabajo, evaluación y condiciones materiales de los centros educativos, y otras reformas orientadas a mejorar el proceso de enseñanza-aprendizaje por medio de prácticas pedagógicas adecuadas a sus necesidades.

Con respecto a la mejora del proceso de enseñanza-aprendizaje, la evaluación es parte fundamental, pues tanto en la educación de jóvenes y adultos como en la escuela tradicional predominan prácticas docentes centradas en la evaluación sumativa, con énfasis en la calificación, alejadas del objetivo real: el aprendizaje. Aún más, este sistema es legitimado por los propios estudiantes cuando piden "la nota de aprobación”. En este sentido, según Santos (2001), la evaluación tradicional no se utiliza como un instrumento de aprendizaje para el que enseña, sino como un simple modo de comprobación del resultado del esfuerzo realizado por el que aprende.

Un estudio realizado en Chile por Vergara (2012) muestra las concepciones y prácticas evaluativas de docentes que trabajan en centros destacados de Educación Básica según el Sistema Nacional de Evaluación del Desempeño de los Establecimientos Educacionales (SNED). En él se concluye que los docentes no tienen claridad con relación a los principios teóricos que sustentan su práctica evaluativa. Existen discrepancias entre los ámbitos técnico y práctico, específicamente en el diseño y la aplicación de procedimientos e instrumentos 
Proceso de reflexión docente para mejorar prácticas de evaluación del aprendizaje en el contexto de la educación para jóvenes y adultos (EPJA) Juan Ariel Muñoz Olivero, Carolina Pilar Villagra Bravo, Segundo Edgardo Sepúlveda Silva

de evaluación: son evidentes la función sumativa y el carácter calificador. Son escasas las instancias de retroalimentación del proceso evaluativo; la autoevaluación y coevaluación no son formativas y el docente califica según criterios personales.

A nivel internacional y dentro del contexto de la educación para jóvenes y adultos, un estudio publicado por Letelier (2009) muestra las características esenciales que ha tenido la evaluación de los aprendizajes por parte de los docentes a través del tiempo. Entre las conclusiones se destacan la práctica de la evaluación con criterios paternalistas, la aprobación como estrategia de retención, la negociación de las calificaciones y la medición centrada en aprendizajes memorísticos en los cuales el producto posee mayor relevancia que el proceso.

Otro punto importante de la evaluación que se debe tener en cuenta es el de los procesos de reflexión que ayudan a mejorar las prácticas docentes y que adquieren vital relevancia dentro del proceso educativo, debido a que estas condicionan, sin duda, el éxito del aprendizaje de los estudiantes. Como ya se dijo, la forma más habitual de evaluación se centra en su función sumativa. El procedimiento más utilizado es la prueba a la cual debe someterse el estudiante. Esto ha permitido que la evaluación se transforme en un instrumento de refuerzo de la verticalidad en las relaciones profesor-estudiante y, en algunos casos, en una forma autoritaria de ejercer poder. Esta modalidad tradicional de evaluar ha sido un factor de discriminación y selección social que margina, excluye y condena al fracaso escolar a gran número de estudiantes (Castro, Correa \& Lira, 2004).

El desafío entonces es lograr cambiar gradualmente esta forma de evaluar por parte de los docentes, mediante instancias de reflexión pedagógica que involucren al estudiante en su proceso de aprendizaje. Este debe saber qué, cómo y para qué será evaluado, debe comprender las fortalezas y debilidades de su propio proceso y recibir retroalimentación constante para poder mejorar.

Sin duda, el impacto que se produciría al mejorar prácticas evaluativas de los docentes, atingentes al contexto de la educación de jóvenes y adultos, provocaría un aumento en la motivación de los estudiantes por aprender y de los propios docentes por enseñar. El aprendizaje sería el centro de la labor pedagógica, y se dejarían de lado prácticas que continúan arraigadas en la cultura evaluativa de los centros educacionales y que producen desmotivación, desinterés, alientan el fracaso escolar de los estudiantes y desencadenan su deserción, tanto en el sistema educativo tradicional como en el que se establece en la EPJA (Letelier, 2009).

En este sentido, Acuña (2013) identifica las razones de la deserción escolar desde el sistema tradicional hacia la educación de adultos. Este autor señala que esta movilidad se da en un rango de edades que fluctúa entre los 15 y los 19 años. Su conclusión es que una de las razones de la deserción es la falta de motivación de los estudiantes, quienes no comprenden lo que enseñan los docentes. Además, el sistema de EPJA les permitiría obtener una certificación más rápida para terminar sus estudios y tendrían mayor flexibilidad para afrontar problemas de índole social o laboral.

Considerando los antecedentes, este estudio presenta los resultados del proceso de reflexión docente sobre las prácticas de evaluación de aprendizaje en un centro integrado de adultos (CEIA). En términos específicos, se desarrolló un diagnóstico comprensivo, en el que se recogió información desde la perspectiva de los docentes. La reflexión se considera fundamental para iniciar procesos de mejoramiento de la práctica pedagógica, en los que el cuestionamiento, la discusión y la autoevaluación resultan ser elementos básicos en el momento de gestionar un currículum que tenga pertinencia y que constituya una oportunidad para el desarrollo educativo de quienes asisten al sistema EPJA.

\section{Marco teórico}

\section{La reflexión pedagógica}

sobre la práctica docente

La capacidad reflexiva es inherente a todo ser humano, pero existen algunas diferenciaciones que vale la pena mencionar. Por un lado, está la práctica reflexiva espontánea que surge cuando existe algún problema que necesita de solución; por otro, una 
Universidad Pedagógica Nacional

Facultad de Humanidades

práctica más metódica, personal y colectiva que se da en el ámbito profesional, incluso cuando las cosas caminan bien, puesto que este proceso reflexivo persigue una mejora continua del trabajo desempeñado (Perrenoud, 2004).

Dentro del contexto de la reflexión como elemento de mejora, en la pedagogía existe un movimiento denominado "investigación-acción”, cuyo propósito fundamental consiste en mejorar la práctica docente y no en generar conocimientos científicos. La utilidad de este movimiento permite que los docentes analicen interacciones, critiquen creencias, teorías, además de evaluar alternativas que conduzcan a cambiar una realidad existente. Este tipo de investigación reflexiva se caracteriza por ser abierta, participativa, democrática y se fundamenta en la mejora de la enseñanza considerando los problemas concretos de los docentes, que conducen a la necesidad de innovar y generar cambios (Blázquez, 1991; Elliott, 1993).

Cuando se está frente al concepto de práctica docente podríamos decir que son todas las acciones pedagógicas que el profesor realiza en el aula, pero esta definición es débil y limitada si pensamos que no solo involucra elementos técnicos propios de la profesión, también se considera al docente como persona que siente, piensa e interactúa en un determinado contexto social.

La práctica docente es de carácter social, objetiva e intencional. En ella intervienen los significados, percepciones y acciones de las personas involucradas en el proceso educativo (alumnos, docentes, padres, autoridades, entre otros). También intervienen los aspectos político-institucionales, administrativos y normativos, que en virtud del proyecto educativo de cada centro delimitan el rol del profesor. Es decir, la práctica docente supone una diversa gama de relaciones entre personas.

La relación educativa con los alumnos es el vínculo fundamental, alrededor del cual se establecen nexos con otras personas: los padres de familia, los demás maestros, las autoridades escolares y la comunidad en general. Se desarrolla dentro de un contexto social, económico, político y cultural que influye en su trabajo, determinando demandas y desafíos (Fierro, Fortoul \& Rosas, 1999). El desempeño del educador está situado en el punto de equilibrio entre el sistema educacional imperante y los grupos sociales particulares que debe atender (Rojas, 2012).

Todas las dimensiones de la práctica pedagógica confluyen en la formación y el desarrollo profesional que el profesor va adquiriendo a través del tiempo. Cabe destacar la capacidad que debe tener el docente de reconocer las fortalezas y debilidades de su labor diaria y potenciar sus competencias por medio de la actualización y formación continua.

Por lo tanto, la mejora de una práctica se refiere no solamente a lo técnico sino también a su concepción axiológica y social, que involucraría tanto los procesos como los resultados finales. Este tipo de reflexión simultánea sobre la relación entre procesos y productos en circunstancias concretas corresponde a lo que se ha llamado práctica reflexiva (Elliott, 1993). El valor de la práctica reflexiva se juzgaría según la calidad de las regulaciones que permite realizar y según su eficiencia en la identificación y resolución de problemas profesionales (Perrenoud, 2004).

La reflexión también se entiende como un proceso ético y filosófico. En cuanto proceso ético, se orienta hacia la elección de un curso de acción para llevar a la práctica los propios valores. Respecto a su carácter filosófico, implica la interpretación de los valores que se traducirán en la práctica. En este sentido, la reflexión sobre los medios es inseparable de la reflexión sobre los fines (Gorodokin, 2005).

Una característica fundamental de la práctica reflexiva es que no admite una postura en la cual los docentes son concebidos como un grupo de individuos que actúan en forma independiente y aislada, sino como sujetos que, permanentemente, construyen y reconstruyen sus significados al compartir sus reflexiones con otros colegas. Cuando los profesores emprenden una reflexión cooperativa sobre preocupaciones comunes e implican a la comunidad en este proceso, consiguen criticar las estructuras curriculares y evaluativas que subyacen 
Proceso de reflexión docente para mejorar prácticas de evaluación del aprendizaje en el contexto de la educación para jóvenes y adultos (EPJA) Juan Ariel Muñoz Olivero, Carolina Pilar Villagra Bravo, Segundo Edgardo Sepúlveda Silva

a sus prácticas y logran la convicción y fuerza necesarias para generar cambios dentro del sistema que las sustenta (Elliott, 1993; Gómez, 2008).

\section{Evaluación de los aprendizajes}

Desde la conceptualización del currículo existen miradas teóricas diversas, que en definitiva determinan los enfoques que se le dan a la evaluación. Iniciamos el análisis distinguiendo dos dimensiones, que están presentes en la práctica pedagógica pero que obedecen a racionalidades teóricas absolutamente opuestas. De acuerdo con Santos (1998a), la evaluación se puede entender desde dos funciones, que responden a dos dimensiones distintas: la evaluación como medición (dimensión tecnológica positivista) y la evaluación como comprensión (dimensión crítica-reflexiva).

Si nos centramos en la evaluación como medición dentro de la dimensión tecnológica positivista, esta es un constructo social y cumple funciones que interesan a unos y perjudican a otros. Al respecto, Santos (1998a) plantea que el proceso de evaluación encierra mecanismos de poder que ejercen el profesor y la institución. Quien tiene capacidad de evaluar, establece los criterios, los aplica de esa forma e interpreta y atribuye causas y decide cuáles han de ser los cambios. Dentro de esta misma postura de la evaluación como medición se reafirma el concepto evaluativo en una enseñanza transmisionista y tradicional en la que se mide el final de un proceso totalmente externo a la enseñanza misma y en la que el estudiante es el principal responsable de su éxito o fracaso, ya que el evaluado es él y no su docente (Smitter, 2006).

Según Zamora y Moreno (2009) la naturaleza de este tipo de evaluación centrada en la medición está dada, fundamentalmente, por la comprobación de los resultados del aprendizaje en el ámbito de los conocimientos. Se lleva a cabo a través de pruebas estandarizadas, iguales para todos, aplicadas en el mismo tiempo y revisadas con criterios similares, lo que lleva a los docentes a centrar su trabajo en una función sumativa y fiscalizadora de la evaluación. Esta concepción favorece y acentúa funciones como el control, la selección, la comprobación, la clasificación y acreditación. Además, esta jerarquización ha traído consecuencias culturales, que se manifiestan en formas específicas como el individualismo, la competitividad, la cuantificación, entre otras (Santos, 1998a).

Esta perspectiva de la evaluación favorece, también, prácticas de enseñanza y de evaluación poco democráticas, puesto que pocas veces se negocian los contenidos, los criterios o su aplicación. Esto hace que los alumnos tengan que moldearse a las exigencias de cada profesor.

Ahora bien, si observamos la evaluación desde la otra vereda, es decir, como comprensión desde la dimensión crítica-reflexiva se puede decir que se entiende como un proceso y no como un momento final, el énfasis está en una evaluación formativa en conjunto con la sumativa (García, 2011). El planteamiento esencial desde esta perspectiva se refiere a la comprensión que se deriva del proceso de análisis. La evaluación no es un momento final del proceso en el que se comprueba cuáles han sido los resultados del trabajo. Es un permanente proceso reflexivo apoyado en evidencias de diverso tipo (Costamagna, 2008).

Según Santos (1998b), en esta perspectiva se favorecen funciones de la evaluación como: diagnóstico, diálogo, comprensión, retroalimentación, mejora y aprendizaje. Desde la mirada propuesta cabe destacar la evaluación vista como un proceso de mejora que no se cierra en sí misma, sino como un abanico de posibilidades que involucra no solo los resultados de un proceso sino también el desarrollo de las prácticas educativas.

Es importante resaltar que el camino para lograr esta mejora involucra acciones que en cierto modo pueden causar desacomodo en los docentes. Por ejemplo, tradicionalmente se piensa que ellos son los únicos capacitados para ejercer procesos de evaluación, por lo tanto, compartir en el aula el juicio evaluativo puede resultar algo complejo. En este sentido Pimienta (2008) asevera que todos los implicados en un proceso educativo podrían evaluar y ser evaluados. La evaluación no es tarea 
Universidad Pedagógica Nacional

Facultad de Humanidades

exclusiva de quien desempeña un papel particular, ni debe ser un instrumento para dominar o ejercer poder sobre otros, sino un instrumento que pueda utilizar cualquier actor del proceso educativo con el fin de mejorar.

En síntesis, y considerando lo planteado por Santos (2002), en las escuelas se tiende a calificar mucho pero se cambia poco. Porque si la evaluación sirviese para aprender, se evitaría la repetición de los errores y se favorecería la mejora de las prácticas. Si solo sirve para medir, clasificar, seleccionar, entre otras acciones desenfocadas del aprendizaje, las fallas se repiten de forma inexorable.

\section{Evaluar para aprender}

Siguiendo con la idea de que la evaluación mal entendida atenta contra el aprendizaje de los estudiantes es preciso señalar que esta no solo mide los resultados, sino que condiciona qué se enseña y cómo, y muy especialmente qué aprenden los estudiantes y cómo lo hacen. Los docentes en su práctica tienden a separar las actividades de aprendizaje de la evaluación, se centran en el desarrollo de los contenidos y las actividades y generalmente realizan una evaluación una vez terminado el proceso. Dedican poca atención a detectar las dificultades de los estudiantes durante el proceso, a comprender sus posibles causas y a pensar en cómo regularlas (Sanmartí, 2007).

Otra postura interesante y que va en la línea de lo señalado son los principios expuestos por Condemarín y Medina (2000), quienes promueven la evaluación auténtica cuyo sustento es aplicar la evaluación como algo cotidiano, integrado en la actividad de aprendizaje. De acuerdo a esto, la evaluación contribuye a regular el proceso de aprender por medio de instancias de autoevaluación y coevaluación; es decir, permite comprender, retroalimentar y mejorar en sus distintas dimensiones y, en consecuencia, ofrece al profesor y al equipo docente la oportunidad de visualizar y reflexionar sobre el impacto de sus propias prácticas educativas, todo lo cual redundará, especialmente, en el mejoramiento de la calidad de los aprendizajes construidos por los estudiantes.
Por su parte, el Ministerio de Educación de Chile, desde la reforma educativa de los años noventa, ha estado orientando a través de diversos instrumentos la reflexión sobre las prácticas de evaluación de los profesores en el aula, asumiendo que esta "debe estar alineada con el propósito de mejorar el aprendizaje porque es inherente al acto educativo" (Vargas, 1998, p. 7).

Para que la evaluación sea parte del aprendizaje, Sanmartí (2007) orienta con algunas ideas claves, que complementadas con los lineamientos del Mineduc se asume que deben ser parte de una planificación efectiva para enseñar y aprender, con el foco puesto en cómo aprenden los estudiantes. Además, se debe concebir como elemento central en la práctica del aula y como una competencia clave de los docentes; debe ser cuidadosa y expresada en positivo, tiene que considerar la motivación del estudiante, los criterios para evaluar tienen que ser compartidos y promover un compromiso hacia el aprendizaje, debe entregar orientaciones constructivas con el fin de mejorar, generar instancias de autoevaluación y coevaluación y por último dar la oportunidad de aprender a todos los estudiantes y en todas las áreas del quehacer educativo.

\section{La evaluación para el aprendizaje en el contexto de la educación} para jóvenes y adultos

Considerando los lineamientos entregados por el Mineduc, por medio de las orientaciones del Decreto exento n. ${ }^{\circ}$ 2169/2007, el proceso de evaluación no se entiende como algo distinto a lo que ya se ha mencionado. Según la Coordinación Nacional de Normalización de Estudios - organismo dependiente de la División de Educación General del Mineduc (2014) —, la evaluación en la EPJA debería considerar la organización de un plan de trabajo que contemple la evaluación diagnóstica, diferenciada, formativa y acumulativa. 


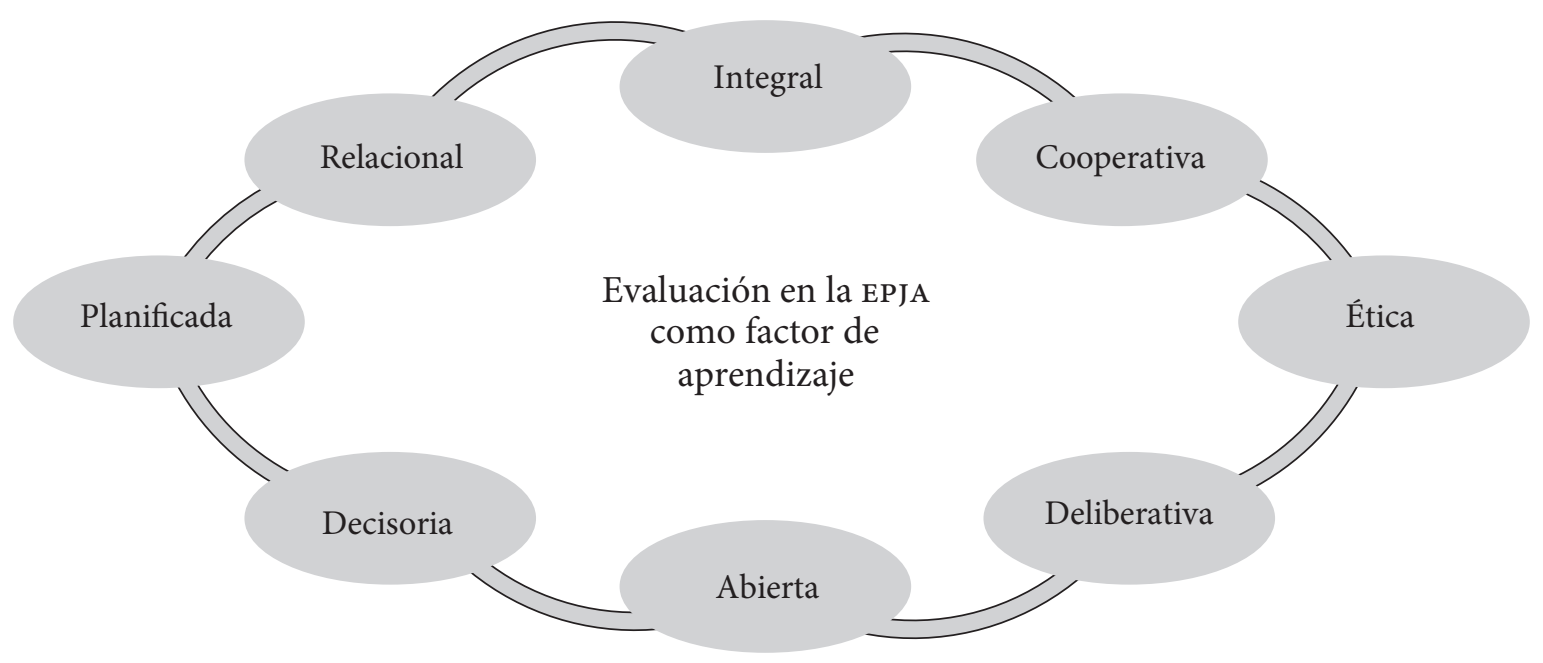

Figura 1. Características de la evaluación en la EPJA.

Fuente: elaboración propia.

La evaluación según el Mineduc (2012), como se observa en la figura 1, es un proceso integral y comprensivo, que proporciona antecedentes sobre la totalidad de variables que intervienen en la enseñanza y el aprendizaje. Es un proceso relacional, es decir, la evaluación informa respecto de cómo aprenden los alumnos y cómo deberían enseñar los docentes. Es un proceso continuo y planificado, que asume un carácter formativo y de retroalimentación para mejorar la calidad de las acciones emprendidas y que van en función del aprendizaje. De carácter cooperativo ya que requiere involucrar a la totalidad de los agentes vinculados al desarrollo del acto educativo (estudiantes y profesores), por medio de la autoevaluación y la coevaluación. Es decisoria cuando proporciona información que permite analizar y adoptar cursos de acción sobre la base de información confiable, lo que aumenta las posibilidades de aprendizaje de los alumnos. Además, tiene un sentido ético que se manifiesta cuando el docente conoce las limitaciones de un alumno y no hace uso de la información disponible para ayudarle a superar sus dificultades. Es deliberativa, ya que implica adoptar las estrategias más apropiadas para conseguir que los alumnos aprendan. Es un factor de aprendizaje, por lo tanto, su propósito solo se logra cuando permite al alumno alcanzar los aprendizajes propuestos y ayuda al centro escolar a lograr su proyecto educativo. Por último, es abierta, ya que aun cuando siga un modelo establecido debe ser capaz de captar e interpretar otros fenómenos significativos que permitan comprender a cabalidad una situación educativa.

\section{Metodología}

Este estudio se realizó en el marco del paradigma cualitativo, pues buscó comprender las suposiciones teóricas respecto de la evaluación de los aprendizajes, que están implícitas en la práctica pedagógica de los profesores que trabajan con jóvenes y adultos en un centro educacional (Páramo y Otálvaro, 2006). Con base en el modelo inductivo se organizó la información emanada desde el discurso de los profesores para develar sus concepciones de evaluación. Los datos permiten comprender el fenómeno en cuestión y orientar las estrategias de mejora a implementar en la formación continua de los docentes. 
El diseño metodológico corresponde a un estudio de caso que consiste en una estrategia de investigación dirigida a comprender las dinámicas presentes en contextos singulares (Martínez, 2006). Es de gran utilidad para el profesorado que participa en la investigación, favorece el trabajo cooperativo, contribuye al desarrollo profesional y por último lleva a la toma de decisiones, a implicarse, a desenmascarar prejuicios o preconcepciones (Latorre, Del Rincón \& Arnal, 2003).

Se utilizaron los grupos de discusión como técnica para la recolección de información. Fue muy útil la generación de espacios de reflexión para conocer las creencias y prácticas evaluativas de los docentes. Se optó por realizar conversaciones cuidadosamente planeadas, diseñadas para un ambiente permisivo, no punitivo (Canales, 2006). Los resultados que se presentan en este artículo corresponden a una primera etapa de sensibilización y reconocimiento (Diagnóstico comprensivo), dentro del proceso de mejora, para continuar en posteriores estudios con la etapa de innovación, para la implementación y consolidación de las prácticas de evaluación de los aprendizajes, tal como se presenta en la figura 2.

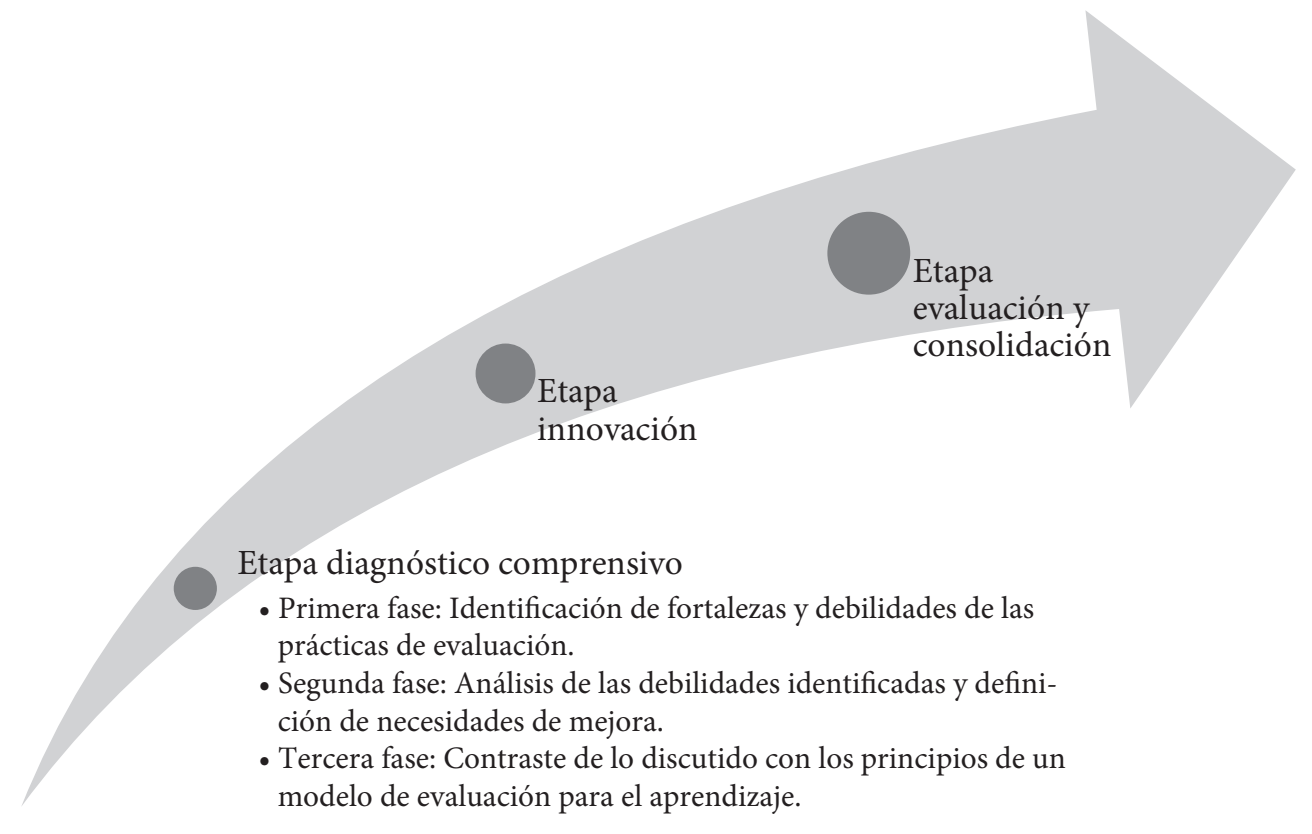

Figura 2. Etapas y fases del proceso de mejora

Fuente: elaboración propia.

Los grupos de discusión se organizaron en tres fases. El propósito de la primera es diagnosticar las prácticas de evaluación que los docentes desarrollan en el aula, identificando fortalezas y debilidades; la segunda fase consiste en analizar las debilidades detectadas en cuanto a las prácticas evaluativas que realizaban como docentes y proponer, basados en su propia reflexión, necesidades de mejora; por último, en la tercera fase, se profundiza sobre las prácticas de evaluación, contrastando lo discutido con un modelo de evaluación que propone ciertos principios y cuya pertinencia en su labor cotidiana analizan los docentes.
Para la sistematización de los resultados se realizó un análisis del discurso de los profesores a nivel manifiesto (Porta \& Silva, 2003), en el cual las preguntas se estructuraron y organizaron para develar sus prácticas de evaluación.

Este estudio se realizó en un centro educativo de adultos que recibe subvención del Estado, con trece años de funcionamiento. Actualmente los estudiantes que atiende se caracterizan por ser de sectores vulnerables, en su gran mayoría desertores del sistema tradicional, jóvenes que trabajan, estudiantes embarazadas y en algunos casos jóvenes 
Proceso de reflexión docente para mejorar prácticas de evaluación del aprendizaje en el contexto de la educación para jóvenes y adultos (EPJA) Juan Ariel Muñoz Olivero, Carolina Pilar Villagra Bravo, Segundo Edgardo Sepúlveda Silva

infractores de ley. El centro atiende en dos jornadas (mañana y tarde) con una matrícula aproximada de 180 alumnos distribuidos en un curso de educación básica $\left(7^{\circ}-8^{\circ}\right)$, seis cursos de enseñanza media, tres de primer nivel $\left(1^{\circ}-2^{\circ}\right)$ y tres de segundo nivel $\left(3^{\circ}-4^{\circ}\right)$. La institución está conformada por una sostenedora, un director, un coordinador pedagógico, siete docentes de aula y dos asistentes de educación.

Los participantes en el estudio son siete docentes, de los cuales seis se desempeñan en la enseñanza media y una docente de enseñanza básica. Sus edades fluctúan entre los 26 y 50 años de edad y el promedio de años de servicio es de once.

\section{Resultados}

A continuación se presenta una síntesis de los principales comentarios realizados por los docentes en función de un guion de preguntas que utilizó un moderador en los grupos de discusión de la primera etapa de diagnóstico, con el propósito de develar, a través de la reflexión colectiva, las prácticas de evaluación de los aprendizajes desde la perspectiva de los profesores.

\section{Resultados de la primera fase: identificación de fortalezas y debilidades}

Todos los docentes manifiestan que las instancias de reflexión sobre temas pedagógicos, específicamente lo que concierne a la evaluación, son escasos. Si bien es cierto, se reúnen en consejo una vez por semana, en general se tratan temas emergentes que en muchas ocasiones no tienen que ver con el ámbito pedagógico; eso sí, destacan que en el último tiempo se ha ido mejorando esa situación por parte de la unidad técnica pedagógica del centro educativo.

Todo el profesorado expresa que el centro educativo no posee un enfoque evaluativo definido, conocido y consensuado, es decir, cada uno evalúa los aprendizajes de sus estudiantes según sus propios criterios. A pesar de que existe un reglamento de evaluación, este solo se consulta para temas administrativos, como número de calificaciones que hay que tener por semestre, plazos para aplicar las evaluaciones, porcentajes de exigencias para casos especiales, entre otros.
Con relación al concepto que tienen de la evaluación de los aprendizajes, los docentes en su mayoría expresan que obedece a una medición de conocimientos de la disciplina, que se realiza por medio de la aplicación de una prueba escrita u oral de carácter sumativo. Una minoría reconoce que realiza evaluaciones más formativas, centradas en la observación directa y la revisión de cuadernos o guías.

Al consultar si la evaluación del aprendizaje que realizan responde a las necesidades de los estudiantes que asisten al centro educativo, las opiniones son divergentes. Una pequeña mayoría explica que las evaluaciones formales (pruebas escritas) les dan resultados; en ellas los estudiantes responden de una u otra manera a esta estrategia, eso sí, con irregulares resultados en sus calificaciones. Otro grupo, también minoritario, plantea que no encuentran la manera de evaluar a los estudiantes, ya que nada los motiva y, por lo tanto, usan la calificación como premio o castigo para lograr algún resultado o una conducta deseada. Por último, un docente expresa que sí logra conectar la evaluación con los intereses de los estudiantes, pero la motivación solo se limita a tener buenas calificaciones.

Un poco más de la mitad de los docentes concuerda en que para evidenciar el logro de los aprendizajes de sus estudiantes usan como procedimientos la prueba escrita, la observación y revisión de cuadernos o guías, los trabajos escritos y en algunos casos exposiciones. Los demás docentes no tienen claro qué es un procedimiento de evaluación. Respecto de los instrumentos, la gran mayoría no los usa para recoger información sobre el aprendizaje de sus estudiantes; solo recurren a la observación directa sin registro escrito, una minoría declara utilizar algunos instrumentos, pero no especifica cuáles.

El profesorado en su totalidad manifiesta que no genera instancias para la autoevaluación y coevaluación. Entre los argumentos entregados, consideran que la evaluación se desvirtúa, los estudiantes no están preparados y tienden a calificarse de manera subjetiva, solo para subir sus notas. Por lo tanto, las opiniones de los estudiantes no estarían siendo consideradas respecto de su proceso de aprendizaje. La mayoría cree que realiza las evaluaciones 
Universidad Pedagógica Nacional

Facultad de Humanidades

con criterios claros y previamente establecidos, sin embargo, no comparten ni informan esta situación al estudiantado. También afirman que la evaluación consiste en las calificaciones parciales que posteriormente se promedian al final del proceso. Del grupo, varios profesores reconocen que solo realizan una retroalimentación de los contenidos tratados al terminar una unidad de aprendizaje.

Una vez descritas las apreciaciones de los docentes sobre su quehacer, se hacen evidentes las debilidades de las prácticas evaluativas. Este primer nivel de reflexión, que obedece a un diagnóstico preliminar, se sintetiza en los puntos siguientes:

- Las instancias de reflexión pedagógica son muy escasas, el tiempo propicio para tal efecto generalmente se utiliza para otras actividades que se alejan de lo pedagógico.

- No existe un enfoque evaluativo definido, conocido y consensuado por los docentes y estudiantes que vaya en función de las necesidades y características particulares de estos últimos, en el contexto de la EPJA.

- Si bien los profesores afirman realizar retroalimentación una vez terminada la unidad de aprendizaje, la descripción de su práctica evidencia que no se involucra al estudiante en su proceso de aprendizaje, no brindan una retroalimentación oportuna, y hay escasas instancias de autoevaluación y coevaluación.

- La evaluación formativa del proceso de aprendizaje es débil. Los docentes tienden a practicar con mayor frecuencia la evaluación final, centrada más en el conocimiento de las disciplinas que en otras dimensiones del aprendizaje, como habilidades y actitudes. Le dan gran importancia a la medición de los aprendizajes evidenciada en una calificación, entendida como la asignación de un número de 1 a 7 según escala chilena.

- Existe una limitación por parte de los docentes en el uso de diferentes procedimientos e instrumentos de evaluación para considerar los diversos estilos y formas de aprendizaje de los estudiantes o bien para medir el logro de los objetivos de aprendizaje del currículo, en el cual los objetivos no solo contemplan la dimensión conceptual de los contenidos.

\section{Resultados de la segunda fase: reflexión sobre las debilidades identificadas}

La segunda fase tuvo como objetivo analizar las debilidades detectadas en cuanto a las prácticas evaluativas que se estaban desarrollando. Se pidió a los docentes que propusieran necesidades de mejora a corto y largo plazo, basados en su reflexión. Los resultados obtenidos fueron los siguientes:

La mayoría del profesorado coincidió en potenciar la función formativa de la evaluación por medio del monitoreo continuo de los aprendizajes y en la generación de mayores espacios de retroalimentación, dentro y fuera del aula. También consideraron la importancia de comenzar a trabajar en la elaboración y el uso de instrumentos de evaluación y generar diversos procedimientos para atender los distintos estilos y formas de aprendizaje. Una minoría creyó necesario discutir y consensuar un enfoque de evaluación que sea atingente a las necesidades de los estudiantes y al contexto del centro escolar, asumiendo que esto implicaría mayor discusión y tiempo.

Hay que destacar que solo dos docentes señalaron como importante involucrar más a los alumnos en su proceso de aprendizaje por medio de la evaluación, y generar los espacios para que ellos se autoevalúen o coevalúen. Por último, solo un docente señaló que es imperioso democratizar la evaluación desarrollando instancias en donde los alumnos evalúen a los docentes (proceso factible en el contexto de la educación de adultos según su opinión). Respecto de estas opiniones, los otros docentes no hicieron ningún comentario.

\section{Resultados de la tercera fase: reflexión sobre las debilidades identificadas}

En la tercera fase se profundizó la reflexión sobre las prácticas de evaluación, complementando y argumentando las conclusiones obtenidas en las 
Proceso de reflexión docente para mejorar prácticas de evaluación del aprendizaje en el contexto de la educación para jóvenes y adultos (EPJA)

Juan Ariel Muñoz Olivero, Carolina Pilar Villagra Bravo, Segundo Edgardo Sepúlveda Silva

fases anteriores, tomando como punto de partida de la discusión un referente teórico-empírico que establece buenas prácticas de evaluación. Durante esta fase los profesores contrastaron lo ya discutido con este modelo, y señalaron cuáles principios serían los más fáciles o difíciles de poner en práctica con el estudiantado.

Existe consenso respecto de la importancia de la evaluación para el aprendizaje. Según el profesorado, esta debería concebirse como un aspecto central en la práctica pedagógica. En este sentido consideran que la evaluación es una de las competencias claves de los profesores, quienes deben poseer la capacidad de observar y detectar las dificultades de los estudiantes para poder ofrecerles retroalimentación oportuna.

Con respecto a la lectura, una minoría del profesorado considera que la evaluación debe obedecer a una planificación efectiva para enseñar y aprender, en la que exista un entendimiento compartido por los estudiantes sobre las metas y criterios para evaluar y que reciban orientaciones constructivas sobre cómo mejorar su aprendizaje. Sustentan este planteamiento argumentando que es responsabilidad profesional del docente planificar y es fácil generar estrategias distintas en esta etapa. Asimismo, manifiestan que es fundamental para el aprendizaje que los alumnos conozcan los criterios con que serán evaluados. Por último, dicen estar de acuerdo con que el docente tenga información precisa de cómo aprenden sus estudiantes para poder guiarlos en su proceso.

En cuanto a los principios que serían difíciles de desarrollar existe mayor consenso del profesorado, quienes consideran que los estudiantes no tendrían la capacidad para autoevaluarse. Opinan que estos procesos generarían instancias para que el estudiante obtuviera poder, lo que visualizan como una amenaza. A juicio de los participantes, el estudiantado no estaría preparado, porque no habría hábitos de estudio, podrían desertar ante situaciones de exigencia, con autoestima baja y con poca capacidad reflexiva y escasa autonomía.
Un grupo minoritario asegura que es complicado que la evaluación considere la importancia de la motivación de los estudiantes, ya que estos no se motivan con nada y, por lo tanto, no aprenderían. Se comenta que les interesa la calificación y no lo que podrían aprender. En esta lógica, explican que sería difícil pretender que la evaluación entregara a los estudiantes orientaciones constructivas sobre cómo mejorar su aprendizaje, los argumentos entregados nuevamente son: la dificultad para detectar fortalezas o debilidades dada la poca motivación de parte de los estudiantes en su aprendizaje.

\section{Discusión}

El proceso de reflexión pedagógica realizado fue la herramienta que se utilizó para determinar las debilidades en las prácticas evaluativas de los docentes y que serán susceptibles de mejora en el futuro, para así potenciar la construcción de aprendizajes del estudiantado en el contexto de la EPJA. Si bien es cierto que los docentes tienen la percepción de que los espacios para la reflexión pedagógica son limitados, al mismo tiempo mencionan que los espacios de discusión generados en este diagnóstico son enriquecedores. Por tales razones el camino seguido parece ser el adecuado. La instancia trabajada obedece a las características de la práctica reflexiva, donde la mejora se desarrolla con foco en los procesos y no solo en los resultados. Este hecho permite cuestionar una situación particular desde dentro del centro escolar, involucrando a las personas responsables de liderar el proceso educativo. La reflexión se caracterizó por ser colectiva y cooperativa; los aportes, visiones y opiniones enriquecieron la discusión para lograr la convicción de mejorar (Elliott, 1993; Perrenoud, 2004).

En cuanto al tema de las prácticas evaluativas de los docentes, ya se mencionó la generación de tres fases de reflexión, que obedecieron a un diagnóstico comprensivo del tema en estudio. Estas instancias aumentaron su nivel de complejidad comenzando por el reconocimiento de la práctica, estableciendo debilidades susceptibles de mejorar, que para 
Universidad Pedagógica Nacional

Facultad de Humanidades

efectos de este estudio corresponden a algunos de los principios de la evaluación desde un enfoque para el aprendizaje.

Una de las debilidades detectadas fue la inexistencia de un enfoque evaluativo definido en el centro educativo. Con relación a este tema, menos de la mitad de los docentes considera prioridad mejorarlo, además no se profundiza por parte del profesorado en la reflexión como un principio relevante. Cabe señalar en este sentido que sí es importante consensuar un enfoque de evaluación que, en este caso, sea pertinente para el contexto del centro escolar y las características del estudiantado. Si se mira la evaluación desde su dimensión críticareflexiva se hace necesario, como lo indica Santos (1998b), que esta cumpla una función dialógica, comprensiva de los procesos y en constante mejora.

Con relación a la participación de los estudiantes en su proceso de aprendizaje, no hay mayor consenso de este como un elemento relevante. Por lo mismo, se requiere discutir y profundizar sobre las responsabilidades que tienen los diversos actores educativos en la evaluación de los aprendizajes, que va más allá de una competencia propia de la función docente. Esto implica cambiar el enfoque en el cual se sustenta el proceso pedagógico. Visto desde un nuevo enfoque, esta postura concuerda con la mirada crítica-reflexiva de la evaluación propuesta por Santos (1998b) y la importancia de la evaluación del proceso, para que el estudiante se haga consciente de su aprendizaje por medio de la autoevaluación, la retroalimentación con sus pares y la del profesor (Pimienta, 2008; Sanmartí, 2007; Smitter, 2006; Zamora \& Moreno, 2009).

La necesidad de modificar el enfoque que subyace a la práctica pedagógica se hace evidente cuando el profesorado manifiesta lo difícil que sería involucrar a los estudiantes en su proceso de aprendizaje para poner en práctica este principio evaluativo. Esto devela el enfoque de transmisión de la enseñanza, hecho que demuestra la necesidad de reflexionar sobre la concepción de aprendizaje escolar para la planificación de acciones de innovación en las prácticas de evaluación de los aprendizajes, considerada como una fase posterior.
En síntesis, el discurso manifiesto de los profesores evidencia la concepción de evaluación tradicional, que considera este proceso como una responsabilidad propia de quien ejerce la función docente. Esta postura es claramente contraria a los nuevos principios de la evaluación para el aprendizaje (Condemarín \& Medina, 2000; Sanmartí, 2007), en la cual se destaca la importancia de la autoevaluación del aprendizaje dentro del aula como algo cotidiano, donde el propio estudiante monitorea y regula su proceso, además de la determinación de metas y criterios de evaluación en conjunto con su profesor.

Con respecto a la evaluación formativa, que es otro de los aspectos débiles por mejorar, se concuerda que es prioridad realizarlas, con mayor monitoreo de los procesos de aprendizaje y con más instancias de retroalimentación. Consideran, además, que debe tener un carácter obligatorio; el monitoreo de los aprendizajes debe hacerse en cada clase, pese a la baja motivación del estudiantado. Estas propuestas son interesantes de analizar, pues pretenden establecerse como normas que se ejecutan en clase, y pierden el sentido formador que tiene en sí misma la evaluación. Por lo mismo, esto evidencia una concepción tradicional de la evaluación, asociada a un ejercicio más técnico y estandarizado que comprensivo.

En cuanto a la motivación, cabe recordar que la calificación por sí sola no es motivante para el aprendizaje. Esta debe ir acompañada de un proceso en el cual el error sea parte del aprendizaje, y tanto los fracasos como los éxitos sean la motivación que debe tener el estudiante para mejorar. El rol del profesor estaría asociado a la generación de condiciones para el aprendizaje (Sanmartí, 2007). Ahora bien, la evaluación centrada en la calificación, en la que importa el resultado y no el proceso, acentúa el control, la selección y la clasificación de los estudiantes y trae como consecuencia una cultura del individualismo, la competitividad y relaciones poco democráticas dentro del aula. Allí, el profesor ejerce dominio sobre el estudiante en lo que respecta al proceso evaluativo (Santos, 1998b). 
Proceso de reflexión docente para mejorar prácticas de evaluación del aprendizaje en el contexto de la educación para jóvenes y adultos (EPJA) Juan Ariel Muñoz Olivero, Carolina Pilar Villagra Bravo, Segundo Edgardo Sepúlveda Silva

\section{Conclusiones}

Con relación al proceso de reflexión pedagógica desarrollado en el presente estudio, se valora la disposición y el interés de los docentes por participar de los distintos grupos de discusión. La temática planteada fue de interés, atingente a un problema real que involucra tanto a profesores como a estudiantes y también al equipo directivo. La práctica reflexiva es responsabilidad de cada uno, pero también es vital que dentro del ámbito de la gestión se den los espacios para que esta se pueda desarrollar de manera colectiva, cooperativa y planificada, con el fin de generar cambios en las prácticas docentes e instalar una cultura de la mejora continua, específicamente en el ámbito de la evaluación que es el motor del aprendizaje (Sanmartí, 2007).

Este estudio demostró que las debilidades expuestas por los profesores con relación a sus prácticas en el área de la evaluación para el aprendizaje en la EPJA no se diferencia de otros estudios hechos a nivel nacional o internacional, tanto de colegios tradicionales que atienden niños, como en el caso de la educación de jóvenes y adultos. En este sentido se repiten concepciones manifestadas en prácticas centradas en la calificación, donde importa más el producto que el proceso de aprendizaje. Sin embargo, lo positivo es que los docentes por medio de estas instancias reflexivas manifiestan el interés de mejorar, lo que facilitará la implementación de innovaciones en sus prácticas de evaluación. En este caso específico, también se potenciarán los aprendizajes de estos jóvenes estudiantes, en su mayoría desertores del sistema tradicional, que como producto de reiterados fracasos escogen esta modalidad de estudios (EPJA) para poder terminar su escolaridad.

Una vez analizados los discursos que se originaron de las debilidades que los propios docentes manifestaron en relación con las prácticas evaluativas se puede concluir que existen divergencias entre el profesorado en lo concerniente a la importancia de determinar un enfoque de evaluación que sustente las prácticas evaluativas en el centro educativo. En este sentido se sugiere seguir trabajando en instancias reflexivas que permitan visualizar la evaluación como una herramienta para la mejora del aprendizaje.

Dentro de los aspectos por mejorar, la mayoría de los docentes considera difícil desarrollar prácticas de retroalimentación durante el proceso de aprendizaje y otorgar a los estudiantes oportunidades para la autoevaluación y coevaluación. Consideran que no es posible hacer un seguimiento del proceso de los estudiantes, debido a la falta de motivación de estos para aprender y su constante irregularidad en la asistencia a clases, este último punto considerado relevante por los docentes. Pese a lo anterior, un pequeño grupo de docentes estima que sí se pueden desarrollar estos procesos ya que se sienten preparados para detectar dificultades y dar retroalimentación a sus estudiantes sobre su proceso de aprendizaje. En cuanto a la autoevaluación y coevaluación, para estos profesores, deben ser prácticas obligatorias en el aula. Lamentablemente, y pese a la intención de mejora, subyace en los discursos un bajo compromiso con el aprendizaje de sus estudiantes. Dan por hecho que la actitud de estos es una causa de lo que ocurre en el aula, de modo que no ven la falta de motivación como una consecuencia de su gestión pedagógica en el aula.

Por último, la mayoría de los docentes estima necesario desarrollar evaluaciones formativas, en este sentido reiteran conceptos de monitoreo constante y retroalimentación. Es interesante destacar que la evaluación formativa se asocia más a momentos del proceso que a su función, que es regular el proceso de enseñanza y aprendizaje. Los desafíos en esta línea se inscriben en trabajar el sentido de la evaluación, no solo como medición, sino como una oportunidad de aprendizaje. Por esto mismo, se requiere ahondar en el significado y uso que se le da a la evaluación, pues el profesorado podría tener dominio en la elaboración de instrumentos y aplicación de procedimientos de evaluación, pero no utilizarlos con un sentido formativo. 
Universidad Pedagógica Nacional

Facultad de Humanidades

\section{Referencias bibliográficas}

Acuña, V. (2013). Abandono de la educación regular de los estudiantes de educación de adultos en Chile. Revista Interamericana de Educación de Adultos, 1, 55-63.

Blázquez, F. (1991). La investigación-acción, Métodos y técnicas de investigación cualitativa. En Sáenz (coord.) Prácticas de enseñanza. Proyectos curriculares y de investigación-acción (pp. 75 -99). Alcoy: Marfil.

Canales, M. (2006). Metodología de investigación social. Introducción a los oficios. Santiago: LOM.

Castro, F., Correa, M. E. \& Lira, H. (2004). Currículum y evaluación. Texto guía. Chillán: Departamento de Ciencias de la Educación, Universidad del Bío-Bío.

Chile, Decreto 2169/2007 Exento, de 07 de noviembre, Ministerio de Educación. Versión única, 24 de diciembre de 2007.

Condemarín, M. \& Medina, A. (2000). Evaluación auténtica de los aprendizajes: un medio para mejorar las competencias en lenguaje y comunicación. Santiago: Andrés Bello.

Costamagna, A. M. (2008). Las prácticas de evaluación de un grupo de profesores de ciencias naturales. Aula Universitaria, 1 (10), 65-74.

Elliott, J. (1993). El cambio educativo desde la investigación-acción. Madrid: Ediciones Morata.

Fierro, C.; Fortoul, B. \& Rosas, L. (1999). Transformando la práctica docente. Una propuesta basada en la investigación acción. México: Paidós.

García, A. (2011, noviembre). Estrategias de evaluación formativa en el aula. Resultados de una investigación nacional en las primarias mexicanas. Trabajo presentado en el xi Congreso Nacional de Investigación Educativa, México. Ponencia recuperada de http:// www.comie.org.mx/congreso/memoriaelectronica/ v11/docs/area_15/0367.pdf

Gorodokin, I. (2005). La formación docente y su relación con la epistemología. Revista Iberoamericana de Educación, 37 (5), 1-9.

Latorre, A.; Del Rincón, D. \& Arnal, J. (2003). Bases metodológicas de la investigación educativa. Barcelona: Experiencia.

Letelier, M. E. (2009). La evaluación de aprendizajes de personas jóvenes y adultas. Revista Iberoamericana de Evaluación Educativa, 2 (1), 147-161.
Martínez, P. (2006). El método de estudio de caso: estrategia metodológica de la investigación científica. Pensamiento \& Gestión, 20, 165-193.

Ministerio de Educación (2012). Material para la elaboración del Reglamento Interno de Evaluación. Santiago: Coordinación Nacional de Normalización de Estudios, División de Educación General.

Ministerio de Educación (2014). Orientaciones para el desarrollo del servicio educativo. Educación para personas jóvenes y adultas (EPJA). Santiago: Coordinación Nacional de Normalización de Estudios, División de Educación General.

Gómez, V. (2008). La práctica reflexiva como estrategia de autoevaluación de las prácticas de enseñanza en los profesores en servicio. Revista Pensamiento Educativo, 43 (2), 271-283.

Osorio, J. (2013). Desafíos docentes en la educación de jóvenes y adultos en Chile: hacia una agenda de conversaciones para el diseño e implementación de nuevas políticas. Revista Temas de Educación, 19 (1), 57-65.

Páramo, P. \& Otálvaro, G. (2006). Investigación alternativa: Por una distinción entre posturas epistemológicas y no entre métodos. Cinta Moebio, 25, 1-7.

Perrenoud, P. (2004). Desarrollar la práctica reflexiva en el oficio de enseñar: profesionalización y razón pedagógica. México: Graó.

Pimienta, J. (2008). Evaluación de los aprendizajes. Un enfoque basado en competencias. México: Pearson Educación.

Porta, L. \& Silva, M. (2003). La investigación cualitativa: el análisis de contenido en la investigación educativa. Buenos Aires: Red Nacional Argentina de Documentación e Información Educativa.

Rojas, M. T. (2012). La investigación acción y la práctica docente. Cuaderno de Educación, 42.

Sanmartí, N. (2007). 10 ideas claves. Evaluar para aprender. Barcelona: Editorial Graó.

Santos, M. A. (1998a). Evaluar es comprender. 2. a ed. Buenos Aires: Magisterio del Río de La Plata.

Santos, M. A. (1998b). La evaluación: un proceso de diálogo, comprensión y mejora. Málaga: Aljibe.

Santos, M. A. (2001). Enseñar o el oficio de aprender. Organización escolar y desarrollo profesional. Rosario: Homo Sapiens.

Santos, M. A. (2002). Una flecha en la diana. La evaluación como aprendizaje. Andalucía Educativa, 34, 7-9. 
Proceso de reflexión docente para mejorar prácticas de evaluación del aprendizaje en el contexto de la educación para jóvenes y adultos (EPJA)

Juan Ariel Muñoz Olivero, Carolina Pilar Villagra Bravo, Segundo Edgardo Sepúlveda Silva

Smitter, Y. (2006). La evaluación de los aprendizajes en una institución formadora de docentes. Revista Educación y Pedagogía, 18 (46), 153-165.

Vargas, C. (1998). Evaluación para aprender: hacia una evaluación participativa en la escuela básica. Santiago: Ministerio de Educación.
Vergara, C. (2012). Concepciones de evaluación del aprendizaje de docentes chilenos destacados de educación básica. Acción Pedagógica, 20 (1), 6-18.

Zamora, M. \& Moreno, T. (2009). La evaluación en las aulas de secundarias. Revista del Centro de Investigación, 8 (32), 99-110. 